\title{
Assessment of Psychophysiological Differences of West Point Cadets and Civilian Controls Immersed within a Virtual Environment
}

\author{
Thomas D. Parsons ${ }^{1, *}$, Christopher Courtney ${ }^{1}$, Louise Cosand ${ }^{1}$, Arvind Iyer ${ }^{1}$, \\ Albert A. Rizzo ${ }^{1}$, and Kelvin $\mathrm{Oie}^{2}$ \\ ${ }^{1}$ University of Southern California's Institute for Creative Technologies. Marina del Rey, \\ CA, USA \\ tparsons@ict.usc.edu \\ ${ }^{2}$ U.S. Army Research Laboratory. Aberdeen Proving Ground, MD, USA
}

\begin{abstract}
An important question for ecologically valid virtual environments is whether cohort characteristics affect immersion. If a method for assessing a certain neurocognitive capacity (e.g. attentional processing) is adapted to a cohort other than the one that was used for the initial normative distribution, data obtained in the new cohort may not be reflective of the neurocognitive capacity in question. We assessed the psychophysiological impact of different levels of immersion upon persons from two cohorts: 1) civilian university students; and 2) West Point Cadets. Cadets were found to have diminished startle eyeblink amplitude compared with civilians, which may reflect that cadets experienced less negative affect during the scenario in general. Further, heart rate data revealed that Cadets had significantly lower heart rates than Civilians in the "low" but not "high" immersion condition. This suggests that "low" immersion conditions may not have the ecological validity necessary to evoke consistent affect across cohorts.
\end{abstract}

Keywords: virtual environment; psychophysiological assessment; immersion; ecological validity, neuropsychology.

\section{Introduction}

Neuropsychological studies tend to assess neurocognitive performance using standardized assessments in controlled settings and behavioral (i.e. self and other) rating scales for assessment of the subject's activities in a real-world setting. While traditional neuropsychological assessments manipulate the complexity of the stimulation, they do little to assess the impact of the intensity of the situation. Neuropsychological assessment should strive for ecologically valid assessments in which findings may reflect the varying levels of intensity found in real world situations. For example, simulations that proffer more intense presentations may elicit accompanying increase in emotional responses. In a related manner, findings from neuropsychological assessments should be generalizable to real-world situations [1]. While controlled

\footnotetext{
${ }^{*}$ Corresponding author.
} 
settings offer increased psychometric rigor, naturalistic (i.e. observation-based) behavioral ratings may have increased ecological validity in that they may better capture the subject's performance in a real world setting. It is important to note that neuropsychological measures in controlled settings and behavioral ratings based upon naturalistic observations do not proffer consistently parallel findings. Further, dissimilar neurocognitive components may be dissociated both by neuropsychological measures in controlled settings and behavioral ratings based upon naturalistic observations [2].

\subsection{Ecological Validity: Verisimilitude and Veridicality}

Current approaches to increasing the ecological validity of neuropsychological assessments reflect the face validity of the assessments and the past experience of the neuropsychologist in drawing inferences between the results and the subject's activities of daily living. There are two approaches to the establishment of ecologically valid neuropsychological assessments: verisimilitude and veridicality [3]. From a human-computer interaction perspective, "verisimilitude" refers to the similarity between task demands of the test and demands imposed in the everyday environment. The establishment of verisimilitude requires "computer" tests that comprise the everyday cognitive tasks of the "human", such that inferences can be easily drawn from computer test results and the human's likely ability to perform those tasks in daily life. Contrariwise, "veridicality" of human-computer interaction refers to the extent to which results on a computer-based assessment are related to scores on other measures (e.g. standardized paper and pencil neuropsychological tests) that predict human performance of real-world tasks [3]. The establishment of veridicality requires that the researcher statistically assess the relationship between human performance on computer-based neuropsychological tests and measures of everyday functioning (e.g. behavioral observations and rating scales).

\subsection{Virtual Environments for Neuropsychological Assessment}

Virtual environments offer the capacity for merging the benefits of controlled settings (e.g. increased psychometric rigor) within ecologically valid virtual environments that simulate the naturalistic environment in which behaviors occur. Recent advances in simulation technology have produced new methods for the creation of virtual environments. With these systems, researchers can present users with ecological verisimilude reflective of "real world" environments. When delivered via a head-mounted display (HMD), an experience of immersion within these captured scenarios can be supported in human users. Immersion has been defined as one's subjective impression that she or he is participating in a comprehensive, realistic experience. Immersion may be enhanced by design strategies that increase the participant's suspension of disbelief [4]. As such, the VR assets that allow for precise stimulus delivery within simulations appear well matched for increasing the ecological validity of neuropsychological assessment.

The value in using virtual reality technology to produce simulations targeting neurocognitive and behavioral applications has been acknowledged by an encouraging body of research. Some of the work in this area has addressed affective processes: anxiety disorders, pain distraction, posttraumatic stress disorder [5]. Other work has 
assessed neurocognitive processes such as attention and executive functioning [6], [7]; memory [8], [9], [10]; and visuospatial abilities [11], [12], [13]. While multiple attempts have been made to apply theoretical perspectives to the development of believable virtual environments, little has been done to "objectively" assess human interpretations of immersion in these environments. There is need for the incorporation of psychophysiological metrics into assessment of human responses while immersed in a virtual environment. As mentioned above, neuropsychological assessment should aim to recreate the environment in which the subject will be processing information. This is especially important when persons are processing information while immersed in environments that have different levels of stimulus intensity. Exposure to emotionally intense situations results in regular activation of cerebral metabolism in brain areas associated with inhibition of maladaptive associative processes [14]. Identical neural circuits have been found to be involved in affective regulation across affective disorders [15], [16]. Systematic and controlled exposure to physiologically intense stimuli may enhance emotional regulation through adjustments of inhibitory processes on the amygdala by the medial prefrontal cortex during exposure and through structural changes in the hippocampus [17].

Thus far, the recording of psychophysiological variables while participants operate within virtual environments has produced useful results in studies examining immersion and presence [18], [19], [20]. As such, the VR assets that allow for precise stimulus delivery within ecologically enhanced scenarios appears well matched for this research. Researchers have found that the individual characteristics of study participants may impact the immersiveness and subsequent findings of a given study. Of primary importance is the extent to which a participant is capable of "absorption" and "hypnotism." Hence, individual differences may moderate presence and confound findings. The propensity of participants to get involved passively in some activity and their ability to concentrate and block out distraction are important factors to consider when conducting a study. Likewise, evidence suggests that hypnotizability plays a role in the outcome of studies using VR. Research into these moderating individual traits is of value because such research may augment participant selection [18].

An important question for ecologically valid virtual environments is whether cohort characteristics affect immersion. If a method for assessing a certain skill is adapted to other cohorts and therefore different for each cohort, data obtained in different cohorts are comparable only when changes to the environment designed for one cohort are not necessary to demonstrate the studied skill in the cohort concerned. For example, neuropsychological assessment of military personnel using a virtual environment that was norm referenced to civilians may not have the same predictive validity for both cohorts. Herein we assessed the psychophysiological impact of different levels of immersion upon persons from two cohorts: 1) civilian university students; and 2) West Point Cadets.

\section{Methods}

\subsection{Participants}

A total of 15 subjects participated in this experiment. Six subjects were West Point cadets and 9 subjects were civilian students and staff at the University of Southern 
California. Strict exclusion criteria were enforced so as to minimize the possible confounding effects of additional factors known to adversely impact a person's ability to process information, including psychiatric (e.g., mental retardation, psychotic disorders, diagnosed learning disabilities, Attention-Deficit/Hyperactivity Disorder, and Bipolar Disorders, as well as substance-related disorders within two years of evaluation) and neurologic (e.g., seizure disorders, closed head injuries with loss of consciousness greater than 15 minutes, and neoplastic diseases) conditions. Subjects were comparable in age, education, ethnicity, sex, and self-reported symptoms of depression.

\subsection{Procedure}

The University of Southern California's Institutional Review Board approved the study. After informed consent was obtained, basic demographic information and computer experience and usage activities were recorded. While experiencing the VRCPAT, participant psychophysiological responses were recorded using the Biopac system. Following completion of the VRCPAT protocol, subjects completed the simulator sickness questionnaire, which includes a pre-VR exposure symptom checklist.

\subsection{Virtual Reality Cognitive Performance Assessment Test}

The project described herein builds upon a larger (ongoing) project that makes use of virtual environments to assess user sensory, perceptual, and neurocognitive performance on various tasks. Neurocognitive and psychophysiological data gleaned from such analyses provides opportunity for implementing systems that can exploit the capabilities of nervous systems, rather than simply depending upon human adaptation, to improve and optimize human-computer interaction. Monitoring the neurocognitive and psychophysiological activity of persons operating within a complex environment, however, poses severe measurement challenges. It is also likely that neurocognitive and psychophysiological responses in operational versus tightly controlled laboratory environments will be significantly, if not fundamentally, different than in controlled laboratory settings.

The VRCPAT project focuses on the refinement of neuropsychological assessment using virtual environments to assess persons immersed in ecologically valid virtual scenarios. The VRCPAT is a three-dimensional virtual environment (i.e. virtual city and Humvee scenarios) designed to run on a Pentium IV notebook computer with one gigabyte RAM and a 128 megabyte graphics card. The primary aim of the VRCPAT project is to use the already existing library of assets as the basis for creating a VE for the standardized assessment of neurocognitive performance within a contextually relevant VE. The application uses USC's FlatWorld Simulation Control Architecture (FSCA). The FSCA enables a network-centric system of client displays driven by a single controller application. The controller application broadcasts user-triggered or scripted-event data to the display client. The real-time three-dimensional scenes are presented using Numerical Design Limited's (NDL's) Gamebryo graphics engine. The content was edited and exported to the engine, using Alias's Maya software. Three-dimensional visual imagery is presented using the eMagin z800. Navigation through the scenario uses a common USB Logitech game pad device. 
Virtual reality-based simulation technology approaches, as delineated herein, are considered to be the future alternative for devising neuropsychological assessment measures that will have better ecological/predictive validity for real-world performance. As well, the flexibility of stimulus delivery and response capture that are fundamental characteristics of such digital environments is viewed as a way for research objectives to be addressed in a more efficient fashion for long term needs. The overall design of this type of assessment tool allows for 1) Verisimilitude: the presentation of realistic environments that reflect activities of daily living; and 2) Veridicality: flexibility in terms of the independent variables that could be studied with this method once the psychometric properties of the standardized test are determined. Such flexibility enables this system to be viewed as an open platform on which a wide range of research questions may be addressed. These include the manipulation of: 1) information load on the front end via the intensity and complexity of target stimuli to be attended to and the type of information in terms of relevance, similarity, vagueness, sensory properties; 2) temporal constraints during varied sustained assessment conditions; 3) distracting activities during the neurocognitive assessments; 4) sensory modality of the information presentation that needs to be attended to; 5) the reward structure used during some tests to assess motivational factors that influence performance; 6) the presentation of aversive stimuli for stressed performance evaluations; and 7) the development of a test bed whereby neurocognitive training and augmented cognition strategies could be assessed under known conditions supported by normative standards.

\subsection{Stimuli and Design}

Subjects were immersed a virtual environment (VE) on two separate experimental runs consisting of both a "high" immersion condition and a "low" immersion condition. In the high immersion condition, subjects wore a head mounted display (HMD) with full tracking capabilities and were free to explore their environment visually. The high immersion condition also made use of headphones and a tactile transducer floor to simulate riding in a large vehicle. The low immersion condition consisted of the same virtual Iraqi scenario presented on a 17 inch laptop screen while wearing headphones. During the low immersion condition, subjects viewed the VE from a static position.

The VE was comprised of a series of safe and combat zones in an Iraqi city. In both the high immersion and low immersion conditions, subjects viewed the VE from the perspective of the driver of a Humvee. The speed and trajectory of the vehicle was kept constant to control for time spent in each zone of the VE. Safe zones consisted mainly of a road surrounded by a desert landscape and were free of gunfire and other loud noises. The combat zones included improvised explosive devices (IEDs), gunfire, insurgents, and screaming voices. Subjects passed through 3 safe and 3 combat zones on each experimental run. The total length of each run was 210 seconds.

An acoustic startle probe was used to elicit startle eyeblink responses. The startle probe was a $110 \mathrm{~dB}$ white noise burst $50 \mathrm{~ms}$ in duration with a near instantaneous rise/fall time presented binaurally through Telephonics TDH-50P headphones. Decibel levels were measured with a Realistic sound level meter using a Quest Electronics earphone coupler. Startle probes were experienced intermittently throughout the 
experimental runs. A total of 4 startle probes were experienced in both the safe and combat zones in each run.

\subsection{Depcholendent Variables}

Psychological Trait Assessment. The following measures were used to assess the impact of absorption and immersiveness upon the "believability" of the system. Prior to the experiment itself, the subjects were required to fill in the following questionnaires: 1) Tellegen Absorption Scale (TAS). The TAS questionnaire aims to measure the subject's openness to absorbing and self-altering experiences. The TAS is a 34item measure of absorption. 2) Immersive tendencies questionnaire (ITQ). The ITQ measure individual differences in the tendencies of persons to experience "presence" in an immersive VE. The majority of the items relate to a person's involvement in common activities. While some items measure immersive tendencies directly, others assess respondents' current fitness or alertness, and others emphasize the user's ability to focus or redirect his or her attention. The ITQ is comprised of 18 items, and each is rated on a 7-point scale.

Psychophysiological Assessment. Psychophysiological assessment included: Electromyographic activity (EMG), Electrodermal activity (EDA), Electrocardiographic activity (ECG), and respiration, which were recorded simultaneously using a Biopac MP150 system and a computer running Acknowledge software.

Startle eyeblink response. EMG startle eyeblink responses were recorded using two small (4mm in diameter) silver-silver chloride electrodes placed over the orbicularis oculi muscle of the left eye and an $8 \mathrm{~mm}$ silver-silver chloride electrode placed behind the left ear to serve as a ground. One $4 \mathrm{~mm}$ electrode was placed directly below the pupil in forward gaze while the other was placed about $1 \mathrm{~cm}$ lateral to the first. The electrodes were placed as close to the eye as possible while still allowing the subject to open and close his or her eyes comfortably. Impedance between the two electrodes was measured and deemed acceptable if below $10 \mathrm{k} \Omega$.

The raw EMG signal was recorded at a rate of $1000 \mathrm{~Hz}$ throughout the experiment using a $10 \mathrm{~Hz}$ high pass and $200 \mathrm{~Hz}$ low pass filter. Raw signals were stored and exported for analysis in microvolt $(\mu \mathrm{V})$ values.

The raw EMG signal was rectified and integrated for analysis. In order to qualify for scoring, the eyeblink trace had to begin within a window of 20 to $100 \mathrm{~ms}$ following the offset of the startle probe. The eyeblink response had to reach peak activity within a window of 20 to $150 \mathrm{~ms}$ following the startle probe. Blinks occurring at longer latencies were not considered to be the result of the startle probe. Amplitudes were recorded as the difference between the peak activity value and the baseline level present immediately preceding onset of the blink response. If the subject was blinking during the onset of the startle probe, that blink response was removed from further analysis due to artifact.

Cardiovascular responding. ECG was recorded with use of a Lead 1 electrode placement, with one $8 \mathrm{~mm}$ silver-silver chloride electrode placed on the right inner forearm about $2 \mathrm{~cm}$ below the elbow and another placed in the same position on the left inner forearm. A third $8 \mathrm{~mm}$ silver-silver chloride electrode was placed on the 
left inner wrist to serve as a ground. Electrode sites were cleaned with alcohol prep pads in order to improve contact. Interbeat intervals (IBIs) were scored as the time difference in seconds between successive R waves in the ECG signal. A median interbeat interval was recorded during each of the same 5 second sampling periods used to assess skin conductance level.

\section{Results}

\subsection{Data Analytic Considerations}

First, we assessed the potential impact of psychological characteristics such as absorption and immersiveness upon the "believability" of the virtual environment. No significant differences were found between groups on these measures. After controlling for potential confounds related to absorption and immersiveness, physiological data were processed using custom-written programs. Within each subject, the median data point for each measure and condition was selected for analyses. Assumptions of a normal distribution equate the mean and median, and the median is less sensitive to outliers. As physiological data are susceptible to introduction of artifact from sources both inside of and outside of the body, the median is equivalent to the mean under ideal circumstances and superior to the mean when artifact alters some data points. Mean-based comparisons between subjects' medians, however, were considered appropriate because artifact-laden data points were already filtered out. A series of ANOVAs were performed on psychophysiological results to assess impact of cohort (Cadet vs Civilian) and level of immersion (High Immersion vs Low Immersion). First analysis of variance was performed on startle eyeblink amplitudes and next upon participants' heart rates, as measured in interbeat intervals.

\subsection{Startle Eyeblink Amplitudes}

A series of ANOVAs were performed. First, a two (Cadet vs Civilian) by two (High Immersion vs Low Immersion) ANOVA was performed on startle eyeblink amplitudes. This analysis revealed a main effect of group membership as Cadets' startle eyeblink amplitudes were smaller than those of Civilians $(\mathrm{F}=7.249, \mathrm{p}<0.05)$. While Cadets had lower startle amplitudes overall, the difference was more exaggerated in the High Immersion condition $(\mathrm{F}=4.695, \mathrm{p}<0.05)$. As increased startle eyeblink amplitude is associated with negative affect, these data suggest that Cadets were less emotionally impacted by the experience than were Civilians.

\subsection{Heart Rates Measured in Interbeat Intervals}

Participants' heart rates, as measured in interbeat intervals, were also analyzed using a two (Cadet vs Civilian) by two (High Immersion vs Low Immersion) ANOVA. When comparing interbeat intervals between groups a strong main effect occurs in the Low Immersion condition. In the Low Immersion condition, Cadets show significantly larger interbeat intervals, meaning a lower heart rate, than Civilians $(\mathrm{F}=17.662, \mathrm{p}<$ 0.05). The effect was not seen for the High Immersion condition $(F=0.001, p=$ 0.997), suggesting that both Cadets and Civilians were responding to the scenario in a similar way. 


\section{Discussion}

Neuropsychological assessment should strive for ecologically valid assessments in which findings reflect the varying levels of intensity found in real world situations. While controlled settings offer increased psychometric rigor, naturalistic behavioral ratings may have increased ecological validity. Unfortunately, neuropsychological measures in controlled settings and behavioral ratings based upon naturalistic observations do not proffer consistently parallel findings. Although virtual environments offer the capacity for merging the benefits of controlled settings (e.g. increased psychometric rigor) within ecologically valid virtual environments that simulate the naturalistic environment in which behaviors occur, the question of whether cohort characteristics affect immersion remains to be established. Herein we assessed the psychophysiological impact of different levels of immersion upon persons from two cohorts: 1) civilian university students; and 2) West Point Cadets.

West Point Cadets were found to have diminished startle eyeblink amplitude compared with civilian controls. As eyeblink amplitude is thought to increase as negative affect increases, so the cadets' relatively small startle eyeblink responses suggest that cadets experienced less negative affect during the scenario in general. For example, there were not significant differences in the Cadets' eyeblinks for level of immersion (i.e. high versus low) or level of scenario intensity (i.e. safe versus ambush zones). Hence, it was difficult to differentiate the impact of immersion upon both cohorts. For both the low and high immersion conditions, Civilians' eyeblink amplitudes were significantly greater than that of Cadets, suggesting that Cadets were well accustomed to experiences with high levels of intensity.

The possibility exists, however, that these data may be interpreted in terms of attention. The startle reflex is often modulated by attentional processing, with more focused attention correlating with a decrease in startle eyeblink amplitude. Findings within the Cadet cohort may reflect their more intense focus on the task, which may have lead to decreased startle responses. Further, it is important to note that startle eyeblink amplitudes in this study were most likely modulated by both negative affect and attention. Unfortunately, the current design does not disentangle the unique contributions of each. Future studies should make use of an attentional processing paradigm to support the differentiation of these components. Cardiac data, however, corroborates the interpretation that negative affect is an important source of variance between Cadets and Civilians.

Heart rate data, collected using interbeat intervals, found that Cadets had significantly lower heart rates than Civilians in the low immersion condition. In the high Immersion condition, however, Cadets' cardiac responses resembled those of Civilians. This suggests that low immersion was insufficient to evoke negative affect in Cadets, but that high immersion impacted the Cadets' affective system. These data also serve to support the affective, versus attentional, interpretation of the startle eyeblink data. If attention were modulating responses there would be equivalent heart rate between the two levels of immersion or a drop in heart rate during high immersion because high immersion engages more attention. The psychophysiological findings of this study suggest that level of immersion is important for Cadet training in order to impact the Cadets' affective system. 
In summary, ecologically valid virtual environments may require increased attention to cohort characteristics. If a method for assessing a certain neurocognitive capacity (e.g. attentional processing) is adapted to a cohort other than the one that was used for the initial normative distribution, data obtained in the new cohort may not be reflective of the neurocognitive capacity in question. The psychophysiological impact of different levels of immersion upon persons from military and civilian cohorts revealed cohort differences suggesting that "low" immersion conditions may not have the ecological validity necessary to evoke consistent affect across cohorts. It is important to note that psychophysiological results in this study were most likely modulated by attentional processing. Unfortunately, the current design does not disentangle the unique contributions of attentional processing. Future studies should make use of an attentional processing paradigm in a larger sample to support the differentiation of these components.

\section{References}

1. Foa, E.B., Kozak, M.J.: Emotional processing of fear: exposure to corrective information. Psychological Bulletin 99, 20-35 (1986)

2. Gordon, M., Barkley, R.A., Lovett, B.J.: Tests and observational measures. In: Barkley, R.A. (ed.) Attention-deficit hyperactivity disorder: A handbook for diagnosis and treatment, 3rd edn., Guilford, New York, pp. 369-388 (2006)

3. Spooner, D.M., Pachana, N.A.: Ecological validity in neuropsychological assessment: A case for greater consideration in research with neurologically intact populations. Archives of Clinical Neuropsychology 21, 327-337 (2006)

4. Dede, C.: Immersive Interfaces for Engagement and Learning. Science 323, 66-69 (2009)

5. Parsons, T.D., Rizzo, A.A.: Affective Outcomes of Virtual Reality Exposure Therapy for Anxiety and Specific Phobias: A Meta-Analysis. Journal of Behavior Therapy and Experimental Psychiatry 39, 250-261 (2008)

6. Parsons, T.D., Bowerly, T., Buckwalter, J.G., Rizzo, A.A.: A controlled clinical comparison of attention performance in children with ADHD in a virtual reality classroom compared to standard neuropsychological methods. Child Neuropsychology 13, 363-381 (2007)

7. Parsons, T.D., Rizzo, A.A.: Neuropsychological Assessment of Attentional Processing using Virtual Reality. Annual Review of CyberTherapy and Telemedicine 6, $23-28$ (2008)

8. Parsons, T.D., Rizzo, A.A., Bamattre, J., Brennan, J.: Virtual Reality Cognitive Performance Assessment Test. Annual Review of CyberTherapy and Telemedicine 5, 163-171 (2007)

9. Parsons, T.D., Rizzo, A.A.: Initial Validation of a Virtual Environment for Assessment of Memory Functioning: Virtual Reality Cognitive Performance Assessment Test. Cyberpsychology and Behavior 11, 17-25 (2008)

10. Parsons, T.D., Silva, T.M., Pair, J., Rizzo, A.A.: A Virtual Environment for Assessment of Neurocognitive Functioning: Virtual Reality Cognitive Performance Assessment Test. Studies in Health Technology and Informatics 132, 351-356 (2008)

11. Parsons, T.D., Larson, P., Kratz, K., Thiebaux, M., Bluestein, B., Buckwalter, J.G., Rizzo, A.A.: Sex differences in mental rotation and spatial rotation in a virtual environment. Neuropsychologia 42, 555-562 (2004) 
12. Parsons, T.D., Rizzo, A.A., Buckwalter, J.G.: Backpropagation and regression: comparative utility for neuropsychologists. Journal of Clinical and Experimental Neuropsychology 26, 95-104 (2004)

13. Parsons, T.D., Rizzo, A.A., van der Zaag, C., McGee, J.S., Buckwalter, J.G.: Gender and cognitive performance: a test of the common cause hypothesis. Aging, Neuropsychology, and Cognition 12, 78-88 (2005)

14. Schwartz, J.M.: Neuroanatomical aspects of cognitive-behavioural therapy response in obsessivecompulsive disorder. An evolving perspective on brain and behaviour. British Journal of Psychiatry Supplemental, 38-44 (1998)

15. De Raedt, R.: Does neuroscience hold promise for the further development of behavior therapy? The case of emotional change after exposure in anxiety and depression. Scandinavian Journal of Psychology 47, 225-236 (2006)

16. Mineka, S., Watson, D., Clark, L.A.: Comorbidity of anxiety and unipolar mood disorders. Annual Review of Psychology 49, 377-412 (1998)

17. Hariri, A.R., Bookheimer, S.Y., Mazziotta, J.C.: Modulating emotional responses: effects of a neocortical network on the limbic system. Neuroreport 11, 43-48 (2000)

18. Macedonio, M., Parsons, T.D., Rizzo, A.A.: Immersiveness and Physiological Arousal within Panoramic Video-based Virtual Reality. Cyberpsychology and Behavior 10, 508 516 (2007)

19. Meehan, M., Insko, B., Whitton, M., Brooks, F.: Physiological measures of presence in virtual environments. In: Proceedings of 4th Annual Presence Workshop, Philadelphia (May 2002)

20. Pugnetti, L., Meehan, M., Mendozzi, L.: Psychophysiological correlates of virtual reality: a review. Presence 10, 384-400 (2001) 